\title{
Clinical and angiographic outcomes after combined direct and indirect bypass in adult patients with moyamoya disease: A retrospective study of 76 procedures
}

\author{
JINBING ZHAO, HONGYI LIU, YUANJIE ZOU, WENBIN ZHANG and SHENGXUE HE
}

Department of Neurosurgery, The Affiliated Brain Hospital of Nanjing Medical University, Nanjing, Jiangsu 210029, P.R. China

Received October 3, 2017; Accepted January 2, 2018

DOI: $10.3892 / \mathrm{etm} .2018 .5850$

\begin{abstract}
The present retrospective study was performed to evaluate the clinical outcome, as well as post-operative collateral formation and revascularization patterns in combined bypass. Surgical revascularization has been the mainstay of treatment for moyamoya patients. A total of 76 hemispheres from 64 moyamoya patients undergoing combined superficial temporal artery-middle cerebral artery (STA-MCA) anastomosis and encephalo-duro-myo-synangiosis (EDMS) were retrospectively reviewed. Computed tomography perfusion and DSA were routinely performed. Modified Rankin scale (mRS) scoring and Kaplan-Meier analysis were performed, and post-operative collateral grading on digital subtraction angiography (DSA), reconstruction patterns and vessel diameter were measured to evaluate the revascularization area and vascular compensatory effect. During the follow-up period, the chief complaint was partially alleviated in 57/64 patients (89.1\%). In all patients, mRS was significantly decreased after the operation. Good revascularization was established in most patients at 6 months after the surgery: $92.1 \%$ of sides were scored as 2 and $77.6 \%$ were scored as 3 by post-operative collateral grading on DSA. Dominant collateral revascularization originating from EDMS was more frequent than that from STA-MCA anastomosis (31 vs. 19 hemispheres) at 6-month follow-up. Morphologically, the calibers of the STA main trunk $(2.60 \pm 0.65 \mathrm{~mm})$ increased profoundly at the first 10 post-operative days $(3.32 \pm 1.05 \mathrm{~mm})$ and shrank
\end{abstract}

Correspondence to: $\mathrm{Dr}$ Shengxue He, Department of Neurosurgery, The Affiliated Brain Hospital of Nanjing Medical University, 264 Guangzhou Road, Nanjing, Jiangsu 210029, P.R. China

E-mail: heshengxue77@126.com

Abbreviations: CTP, computed tomography perfusion; DSA, digital subtraction angiography; DTA, deep temporal artery; EDMS, encephalo-duro-myo-synangiosis; MMA, middle meningeal artery; MMD, moyamoya disease; mRS, modified Rankin scale; STA-MCA, superficial temporal artery-middle cerebral artery

Key words: moyamoya disease, direct bypass, indirect bypass, revascularization, collateral formation back to pre-operative status at 6 months $(2.20 \pm 1.01 \mathrm{~mm})$ and 12 months $(2.36 \pm 0.73 \mathrm{~mm})$ according to DSA examination. Middle meningeal artery (MMA) and deep temporal artery (DTA) demonstrated continuous augmentation during the follow-up time (from $1.30 \pm 0.46$ to $1.87 \pm 0.69 \mathrm{~mm}$ for MMA and $1.11 \pm 0.25$ to $2.11 \pm 1.16 \mathrm{~mm}$ for DTA). These results strongly suggested that combined STA-MCA bypass and EDMS provided efficient revascularization and reduced acute cerebral events. The direct STA-MCA bypass provided early augmentation of cerebral perfusion, whereas the indirect EDMS provided a more durable long-term revascularization, indicating a complementary association between the two revascularization methods.

\section{Introduction}

Moyamoya disease (MMD) is a unique clinical entity that is characterized by the progressive occlusion of the bilateral supraclinoid internal carotid artery. Collateral formation at the base of the brain occurs accordingly to compensate for the insufficient cerebral perfusion. If new-formed collaterals are not sufficient to maintain normal cerebral perfusion, ischemic events occur, particularly in pediatric patients who require more blood flow for cerebral development and maturation. Excessive hemodynamic stress in the collateral circulation may also cause hemorrhages, which mainly occurs in adult patients. The goal of treatment for MMD is to improve cerebral blood flow; however, medical treatments appear to be ineffective in preventing ischemic and hemorrhagic events (1,2). Surgical revascularization represents an optimal therapeutic option (3). Surgical procedures for MMD may be basically classified into three categories: Direct bypass, including superficial temporal artery-middle cerebral artery (STA-MCA) and occipital artery-MCA, indirect bypass, including encephalo-duroarterio-synangiosis, encephalo-myo-synangiosis (EMS), encephalo-duroarterio-myo-synangiosis and encephaloduro-myo-synangiosis (EDMS), and combined bypass. However, the optimal revascularization strategy for symptomatic adult moyamoya patients remains controversial (4). Direct bypass offers the advantage of immediate revascularization, and indirect bypass induces in-growth of collaterals over time. Combined procedures, owing to the advantages of direct and indirect bypasses, appear reasonable, but this requires to be further supported with additional clinical evidence (5-8). To 
date, no large randomized clinical trials have been performed, and the clinical surgical choice is largely dependent on the preference and training experience of the cerebrovascular neurosurgeons.

In the present study, 64 adult moyamoya patients (76 hemispheres) who underwent combined STA-MCA bypass and EDMS at Nanjing Brain Hospital (Nanjing, China) from January 2010 to December 2015 were reviewed. Clinical outcomes, post-operative collateral formation and revascularization patterns, cerebral perfusion and changes in donor vessel caliber were evaluated to facilitate the choice of surgical procedure for MMD in adults.

\section{Materials and methods}

Patients and clinical data. The clinical data of 64 adult moyamoya patients (76 hemispheres) who underwent combined STA-MCA bypass and EDMS at Nanjing Brain Hospital (Nanjing, China) from January 2010 to December 2015 were reviewed. The present retrospective study was approved by the ethics committee of the Brain Hospital affiliated to Nanjing Medical University (Nanjing, China). Written informed consent was provided by the patients or their guardians.

All of the patients had typical MMD as specified by previously published criteria (2). Patients with the characteristic moyamoya vasculopathy but no associated risk factors were diagnosed with MMD, whereas those with generally acknowledged risk factors, including head/neck radiotherapy (particularly for optic gliomas, craniopharyngiomas or pituitary tumors), Down's syndrome, neurofibromatosis type 1, sickle cell anemia, Graves' disease, meningeal infections including tuberculous meningitis were diagnosed with moyamoya syndrome. The detailed medical history of all the patients enrolled in the present study was recorded, based on which all of those patients with well-recognized risks were excluded. The patient inclusion criteria were as follows: i) Age, $>18$ years; ii) clinical diagnosis of MMDs confirmed by DSA; iii) patients were symptomatic or had evidence of cerebral hemodynamic compromise based on computed tomography (CT) perfusion (CTP). Pediatric patients (age, $<18$ years) were excluded.

Surgical procedures. The neurosurgeons at our department are experienced in performing STA-MCA bypass and EDMS operations. After intravenous anesthesia, the respective patient was placed in the supine position with the head rotated $60-80^{\circ}$ toward the opposite side of the surgery. The courses of the STA trunk and branches were marked on the skin, and a frontal-temporal-parietal incision was designed accordingly. The skin flap was dissected between the galea aponeurotica and temporal fascia. The temporal muscle was dissected from the bone with a retrograde dissection technique $(9,10)$, and the deep temporal artery (DTA) was easily preserved. A heart-like frontal-temporal bone flap was made along the superior temporal line, and the middle meningeal artery (MMA) passing through the sphenoidal crest was kept intact. The dura mater was incised along the MMA and its major branches, resulting in dura mater strips with a width of $0.5-1.0 \mathrm{~cm}$. The remaining part of the dura mater was separated by radial incisions and flipped over. During this procedure, it is important to preserve the DTA and MMA, as they are the major source for revascularization in EDMS. M4 branches on the cortical surface with a diameter of $>0.8 \mathrm{~mm}$ were suitable for direct anastomosis. An adequate length of the STA branch was dissected, trimmed and anastomosed end-to-side to the M4 cortical artery with 10-12 interrupted sutures. The temporal muscle covered the exposed cortex and was fixed on the margin of the bone window. The bone flap was trimmed to an appropriate shape and fixed without compressing the STA. Usually, the total operation time was $\sim 4 \mathrm{~h}$. The average blocking time was $\sim 20 \mathrm{~min}$. Intraoperative blood loss was $\sim 300 \mathrm{ml}$. Anemic moyamoya patients received a blood transfusion during the operation, usually a $2 \mathrm{U}$ red blood cell suspension, to ensure adequate blood volume and avoid low cerebral perfusion.

The post-operative therapy for moyamoya patients at our center includes anti-platelet, anti-oxyradical and blood volume management. The patient is prescribed aspirin $(100 \mathrm{mg}$, quaque nocte) if no obvious intracranial hemorrhage is visible on routine head CT. 2,500-3,000 ml intravenous fluid infusion per day was necessary during the early stage after surgery. Semiliquid and soft diets are preferred for the patients after surgery.

Clinical follow-up and post-operative angiographic evaluation. Post-operative complications were recorded in detail, including motor and sensory deficits, epilepsy and hemorrhage. Neurological deficits and recurrent cerebral vascular events were evaluated by modified Rankin scale (mRS) scoring and Kaplan-Meier analysis. CT, CT angiography (CTA), CTP and DSA were routinely performed; cranial CT and CTA examinations were performed on the first post-operative day and further CTP and DSA examinations were required for all patients at 6 months after surgery. However, not all patients were routinely available during the follow-up period, certain patients underwent DSA on the 10th day following surgery, 12 months following surgery or yearly thereafter.

Pre-operative angiographic stages were classified according to the standards proposed by Suzuki and Takaku (11). Post-operative collateral grading was evaluated by reviewing the lateral view of the external carotid artery angiography. The newly developed collateral circulation through the combined revascularization was graded according to angiographically visible supplied territory dimensions (12). In brief, scoring was as follows: 0, No collaterals present in the target revascularization area; 1 , collaterals present in one-third or less of the MCA territory; 2, in one to two thirds of the MCA territory; 3 , in >two thirds of the MCA territory. This grading method provides an overall evaluation of the compensation area without considering the collaterals derived from the direct bypass or the EDMS. Using these scores, the patients were categorized into 4 patterns of revascularization (13): Pattern I, direct revascularization with a higher score than indirect revascularization; pattern II, direct revascularization with a similar score to indirect revascularization; pattern III, indirect revascularization with a lower score than direct revascularization; pattern IV, no obvious revascularization.

Indirect revascularization mainly depended on the contribution of different donor arteries, including the MMA 
and DTA. Direct revascularization mainly depended on the contribution of the STA. DSA post-processing software (GE Healthcare Advantage workstation 4.0; GE Healthcare Advantage workstation; GE Healthcare, Little Chalfont, UK and Siemens Axiom Artis 3.0; Siemens, Ag, Munich, Germany) were used to measure the inner diameters of the STA trunk, its frontal and parietal branches, MMA and DTA on the lateral angiographic projection of the external carotid artery. The measurement sites were marked as follows (Fig. 1): STA trunk-proximal to its bifurcation into frontal and parietal branches; frontal and parietal branch-proximal to its first bifurcation; MMA-distal trunk after the MMA passing through the spinous foramen, which appears as a sharp angle on angiography; DTA-the trunk distal to the origination from the maxillary artery. Measurements were performed at similar locations on the follow-up angiograms at different time-points (10 days, 6 months and 12 months after surgery). The calculation work was performed by 2 blinded neurointerventionalists.

Statistical analysis. SPSS 17.0 (SPSS, Inc., Chicago, IL, USA) and GraphPad Prism 5.0 (GraphPad Software, Inc., La Jolla, USA) were used for statistical analysis. Differences among different groups were assessed using a Student's t-test (paired sample). Non-parametric data were analyzed by Fisher's exact test. Survival evaluation was performed using Kaplan-Meier analysis. Values are expressed as the mean \pm standard deviation. $\mathrm{P}<0.05$ was considered to indicate a statistically significant difference.

\section{Results}

Patient demographics and clinical presentation. Combined bypass surgery was applied to 76 hemispheres of 64 moyamoya patients from January 2010 to December 2015. The patient cohort comprised 31 males and 33 females aged 20-68 years. The follow-up duration was 6-70 months (mean, 23 \pm 14 months). Of the operated hemispheres, 21 were on the left, 29 on the right and 13 were bilateral. The most common clinical manifestation of MMD in this series was cerebral ischemia, including transient ischemic attack (TIA) and infarction. A total of 42 patients (65.6\%) presented with ischemic symptoms (12 TIA and 30 cerebral infarctions), while $14(21.9 \%)$ patients presented with spontaneous intraventricular or intraparenchymal hemorrhage. Another 8 patients $(12.5 \%)$ complained of headache. All of the patients received a definite diagnosis of MMD and underwent evaluation of cerebral perfusion and vascular compensation prior to surgical interventions by head CT, magnetic resonance scan, CTP and DSA. According to the scale by Suzuki and Takaku (11), 26, 35, 12 and 3 hemispheres were considered to be stage II, III, IV and V, respectively, while none were stage I or VI. Surgical plans were largely dependent on the clinical symptoms, signs and cerebral perfusion of MMD patients. In detail, bypass was performed on the hemisphere with worse cerebral perfusion and collateral compensation first, followed by the contralateral side. If the cerebral perfusion and collateral compensation of bilateral hemispheres were similar, the non-dominant hemisphere was preferred, which was mostly the right-hand side. For patients with hemorrhagic onset, the bleeding hemisphere was prioritized. All 64 MMD patients

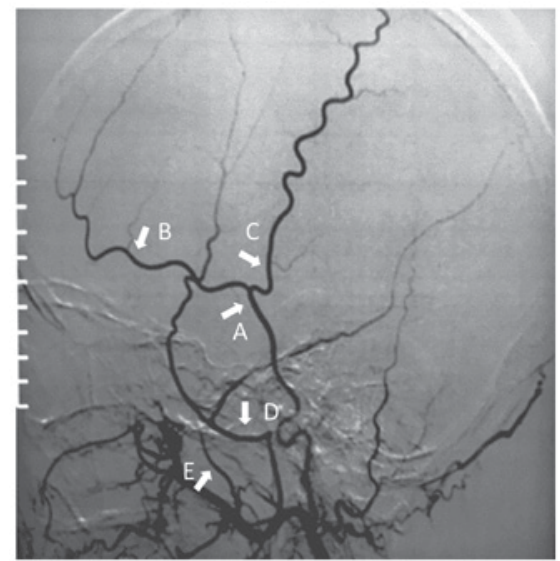

Figure 1. Illustration of the measurement sites of target arteries in the lateral view of selective external carotid angiography. (A) Proximal to the bifurcation of the superficial temporal artery into frontal and parietal branches; (B) proximal to its first bifurcation of the frontal branch; (C) proximal to its first bifurcation of the parietal branch. (D) Distal trunk after the middle meningeal artery passes through the spinous foramen. (E) The deep temporal artery trunk distal to its origination from the maxillary artery. Scale bar, $1 \mathrm{~cm}$.

received combined bypass. The characteristics of enrolled patients are summarized in Table I.

\section{Clinical outcomes for MMD patients}

Peri-operative complications. Post-operative complications frequently occurred during the first two weeks after the operation. Within the cohort, new clinical symptoms in the ipsilateral and contralateral hemisphere appeared in 16/64 patients (25\%). Symptomatic cerebral hyperperfusion syndrome or TIA was observed in nine patients $(14.1 \%)$, who experienced transient numbness of the limbs and/or inarticulate speech without any new parenchymal lesions. A total of four patients $(6.25 \%)$ experienced new-onset cerebral infarction. Transient seizure occurred in two patients $(3.13 \%)$, and one patient $(1.56 \%)$ developed slight cerebral hemorrhage in the ipsilateral basal ganglia.

Neurological improvements during follow-up. Three patients continued to complain of dizziness, headache and limb numbness, respectively. Two patients suffered a TIA and one patient had a huge hematoma in the contralateral hemisphere at 18 months after surgery as a result of sudden unconsciousness. This patient received emergent hematoma removal, decompressive craniectomy and EDMS, and remained in a state of severe disability during the follow-up period. Another patient developed a headache due to slight intraventricular hemorrhage at the 30th post-operative month. No mortalities occurred until the end of the follow up period. mRS scoring was performed to evaluate neurological deficits pre-operatively and at 6 months after the operation. As presented in Fig. 2, the mRS score was significantly decreased after the operation not only in all patients $(1.55 \pm 0.95$ pre-operation vs. $0.76 \pm 1.05$ post-operation, $\mathrm{P}<0.001)$, but also in the ischemic subgroup $(1.45 \pm 0.96$ pre-operation vs. $0.71 \pm 1.01$ post-operation, $\mathrm{P}<0.01)$ and hemorrhagic subgroup $(1.86 \pm 0.77$ pre-operation vs. $1.07 \pm 1.14$ post-operation, $\mathrm{P}<0.05)$. However, no statistically significant difference was present between these two subgroups. Kaplan-Meier analysis was performed, considering the 
Table I. Clinical characteristics of the enrolled 64 moyamoya patients undergoing combined STA-MCA and EDMS.

\begin{tabular}{|c|c|}
\hline Parameter & $\mathrm{N}$ \\
\hline \multicolumn{2}{|l|}{ General characteristics } \\
\hline No. of patients (hemispheres) & $64(76)$ \\
\hline Mean age \pm SD (years) & $42 \pm 11$ \\
\hline Gender (male/female) & $31 / 33$ \\
\hline \multicolumn{2}{|l|}{ Initial presentation } \\
\hline TIA & 12 \\
\hline Infarction & 30 \\
\hline Hemorrhage & 14 \\
\hline Headache & 8 \\
\hline \multicolumn{2}{|l|}{ Suzuki and Takaku stage } \\
\hline I/II/III/IV/V/VI & $0 / 26 / 35 / 12 / 3 / 0$ \\
\hline \multicolumn{2}{|l|}{ Surgical procedures } \\
\hline Hemispheres (left/right/bilateral) & $21 / 29 / 13$ \\
\hline STA-MCA+EDMS & 76 \\
\hline \multicolumn{2}{|l|}{ Postoperative complications } \\
\hline TIA/Hemodynamic disorders & 9 \\
\hline Infarction & 4 \\
\hline Epilepsy & 2 \\
\hline Hemorrhage & 1 \\
\hline \multicolumn{2}{|l|}{ Clinical follow-up } \\
\hline Mean follow-up period \pm SD (months) & $23 \pm 14$ \\
\hline Symptoms (improved/no change/aggravated) & $57 / 3 / 4$ \\
\hline Re-hemorrhage, $n$ & 2 \\
\hline \multicolumn{2}{|l|}{ Postoperative angiographic grading } \\
\hline$<1 / 3$ MCA territory & 6 \\
\hline 1/3-2/3 MCA territory & 11 \\
\hline$>2 / 3$ MCA territory & 59 \\
\hline
\end{tabular}

Valuesareexpressed asn.STA-MCA,superficial temporal artery-middle cerebral artery anastomosis; EDMS, encephalo-duro-myo-synangiosis; TIA, transient ischemic attack SD, standard deviation.

time-point of ischemic or hemorrhagic events as the end-point. When no such event occurred over the follow-up period, the time recorded was the last available clinical follow-up time (Fig. 3). The overall rate of cerebral ischemia and hemorrhage was 19.7 and $3.9 \%$ respectively.

\section{Angiographic outcomes}

Vascular remodeling characterizations. All of the patients received DSA examination at 6 months after the operation. A total of 3 STAs were occluded. Generally, good revascularization was established in most patients. On post-operative collateral grading, $92.1 \%$ (70/76) of sides were scored as 2 and $77.6 \%(59 / 76)$ of sides were scored as 3. Poor revascularization with sparse vessels was obtained in 6 sides (6 MMD patients), whose distribution area was less than $1 / 3$ of the MCA territory. At 12 months after combined bypass, 40 patients received DSA re-examination. A total of 35 sides (87.5\%) were scored as 2 or 3 . The collateralization area of the direct and indirect

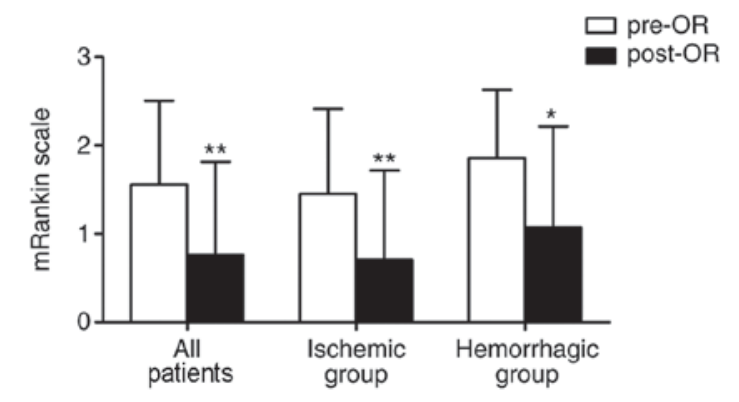

Figure 2. Evaluation of neurological deficits preoperatively and 6 months after OR via the mRS. The mRS score was significantly decreased after the operations in all enrolled patients $(1.55 \pm 0.95$ vs. $0.76 \pm 1.05)$, in the ischemic subgroup ( $1.45 \pm 0.96$ vs. $0.71 \pm 1.01)$ and the hemorrhagic subgroup (pre-OR vs. post-OR: $1.86 \pm 0.77$ vs. $1.07 \pm 1.14$ ). No statistically significant difference was identified between these two subgroups. Values are expressed as the mean \pm standard deviation. ${ }^{*} \mathrm{P}<0.05,{ }^{* *} \mathrm{P}<0.01$ pre- vs. post-OR. mRS, modified Rankin scale; OR, operation.

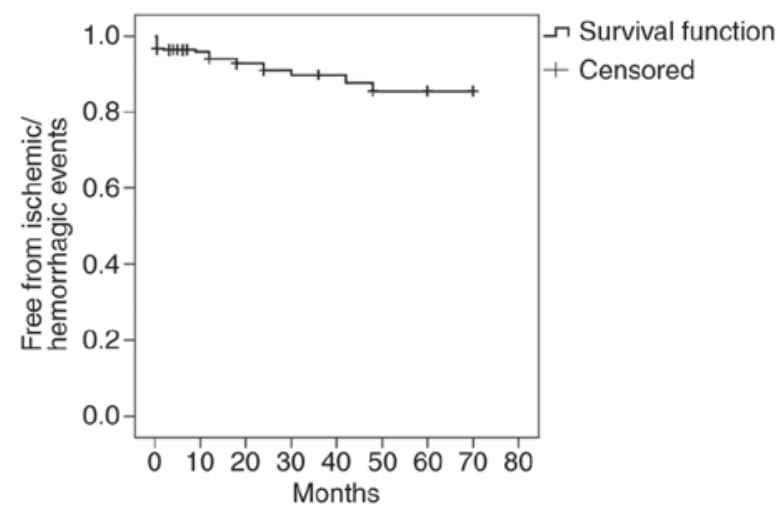

Figure 3. Kaplan-Meier analysis indicated that the majority of patients were free of ischemic and/or hemorrhagic events during the entire follow-up period. Censoring is indicated by the tick marks. The overall rate of cerebral ischemia and hemorrhage was 19.7 and $3.9 \%$, respectively.

bypass was then compared. At 6 months after the operation, 19 hemispheres demonstrated that STA-MCA anastomosis provided a greater collateral territory than EDMS (STA-MCA anastomosis >EDMS, pattern I), while the opposite (STA-MCA anastomosis <EDMS, pattern III) was observed in 31 hemispheres. An equivalent contribution to revascularization (STA-MCA anastomosis=EDMS, pattern II) was present in 25 hemispheres. Pattern IV vascular remodeling was observed in only one hemisphere (Fig. 4).

Morphological alterations of donor vessels. Compared with the pre-operative caliber $(2.60 \pm 0.65 \mathrm{~mm})$, the caliber of the major trunk of the STA increased profoundly at post-operative day $10(3.32 \pm 1.05 \mathrm{~mm}, \mathrm{P}<0.05$ vs. pre-operation), and shrank back to the pre-operative caliber at 6 months $(2.20 \pm 1.01 \mathrm{~mm}$, $\mathrm{P}>0.05)$ and 12 months $(2.36 \pm 0.73 \mathrm{~mm}, \mathrm{P}>0.05)$. On DSA, STA shrinkage occurred in parallel with the development of prominent collaterals from indirect bypass at 6 months post operation, indicating that the STA may have a dominant role through providing immediate flow augmentation in the early phase after surgery and may slowly regress due to indirect neoangiogenesis and revascularization. Longitudinal changes (pre-operation vs. 10 days vs. 6 months post-surgery) 


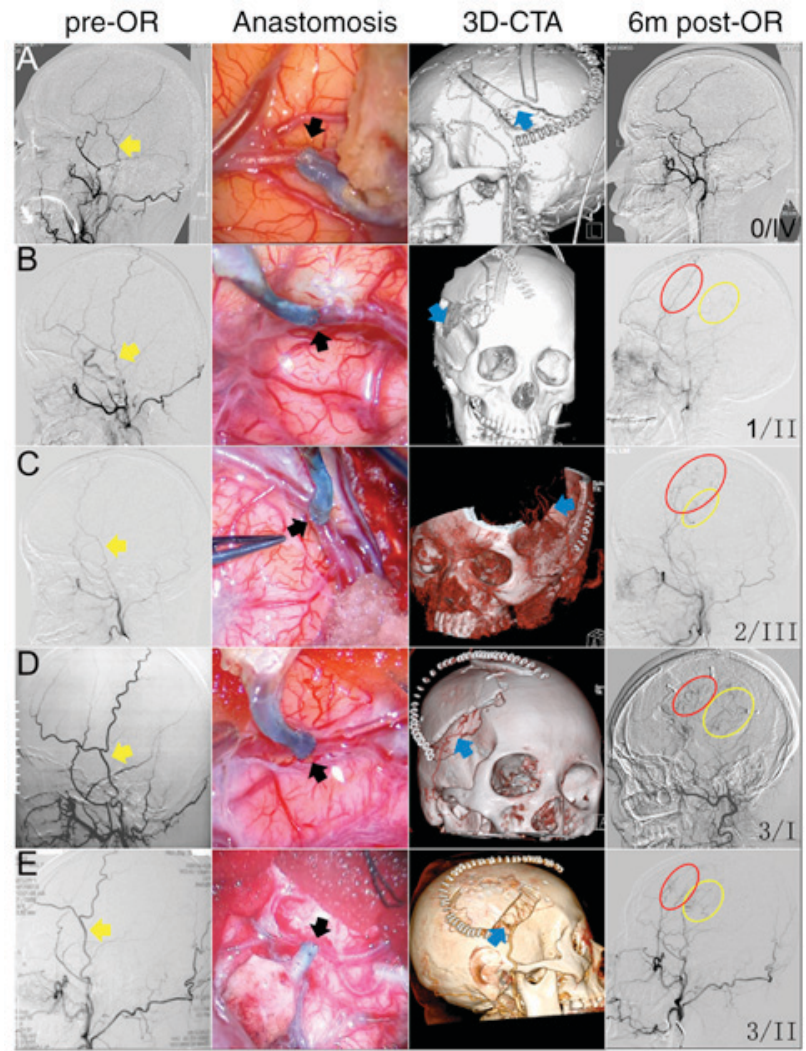

Figure 4. (A-E) Typical images from five different moyamoya patients, respectively, during the peri-operative and follow-up examinations. The first column displays the lateral view of the external carotid artery angiography, where the yellow arrow indicates STA, the second column presents the intraoperative status of the anastomosis between STA and MCA (black arrow); 3D-CTA indicated the STA branch extending from the temporal bone window into the intracranial space (blue arrow) in the third column; images in the fourth column present different postoperative collateral grading scores and reconstruction patterns on DSA. The red circles indicate the position collateral from the indirect bypass (encephalo-duro-myo-synangiosis) and the yellow circles indicate the site collateral from the direct bypass (STA-MCA anastomosis). Postoperative collateral grading on DSA and reconstruction patterns are indicated on the right-lower corner. STA-MCA superficial temporal artery-middle cerebral artery; DSA, digital subtraction angiography; OR, operation; 3D-CTA, three-dimensional reconstructive computed tomography angiography.

of the frontal and parietal branches of the STA were similar to those of the STA trunk; however, they did not achieve statistical significance (frontal branch: 1.48 $\pm 0.47,2.01 \pm 0.59$ and $1.31 \pm 0.44 \mathrm{~mm}$; parietal branch: $1.24 \pm 0.47,1.87 \pm 1.10$ and $1.50 \pm 0.75 \mathrm{~mm})$. The MMA and DTA are the major donor vessels in EDMS. Over the observation period, the diameter of MMA was obviously enlarged at 10 days $(1.72 \pm 0.54 \mathrm{~mm}$, $\mathrm{P}<0.05$ vs. pre-operation), 6 months $(1.92 \pm 0.79 \mathrm{~mm}, \mathrm{P}<0.05)$ and 12 months $(1.87 \pm 0.69 \mathrm{~mm}, \mathrm{P}<0.05)$ in comparison to the pre-operative diameter $(1.30 \pm 0.46 \mathrm{~mm})$. Similar morphological changes were observed in the DTA whose diameter significantly increased from $1.11 \pm 0.25 \mathrm{~mm}$ (pre-operation) to $2.02 \pm 0.47 \mathrm{~mm}$ at post-operative day $10,2.13 \pm 0.52 \mathrm{~mm}$ at 6 months and $2.11 \pm 1.16 \mathrm{~mm}$ at 12 months $(\mathrm{P}<0.05$ vs. pre-operation for each). This compensative enlargement was probably due to increased blood flow after indirect bypass.

Cerebral perfusion after surgery. CTP was applied to evaluate cerebral blood supply on admission and at 6 months

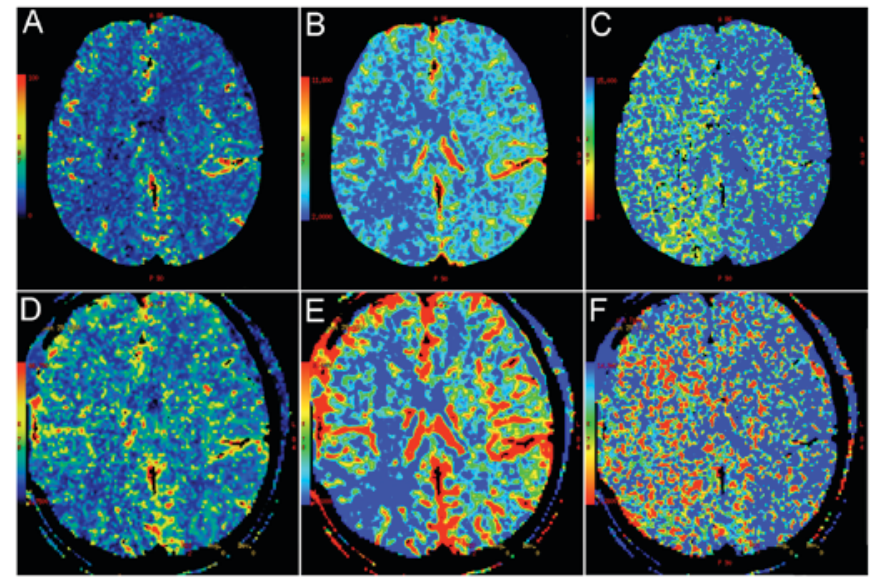

Figure 5. Cerebral blood supply change on computed tomography perfusion imaging (A-C) on admission and (D-F) 6 months after the operation of the right hemisphere. (A and D) Mean blood flow and (B and E) mean blood volume were enhanced on the operated side when compared to the contralateral side, while ( $\mathrm{C}$ and $\mathrm{F}$ ) the mean transit time was shortened after the operation.

after the operation. At 6 months, 70 hemispheres $(92.1 \%$; 58 MMD patients) demonstrated increased cerebral blood flow compared with the pre-operative one. As presented in Fig. 5, the mean blood flow and mean blood volume were enhanced in the operated side compared with that in the other side, while the mean transit time was shortened after the operation.

Vascular reconstruction discrepancies between ischemic and hemorrhagic patients. Of the enrolled patients, 42 (65.6\%, 49 hemispheres) presented with ischemic symptoms, while 14 patients $(21.9 \%, 17$ hemispheres) presented with hemorrhage. At 6 months, in the ischemic group, the number of patients with an angiographic collateralization score of $0,1,2$ and 3 was $0,3,6$ and 40 respectively, which is similar to that in the hemorrhagic group (0, 1, 3 and 13) according to Fisher's exact test. In addition, the contributions from EDMS or STA-MCA anastomosis to re-vascularization were similar (Table II). Thus, patients in the ischemic and hemorrhagic group had similar angiographic collateralization score and vascular reconstruction patterns.

\section{Discussion}

Within the cohort of the present study, most moyamoya patients recovered well after receiving combined STA-MCA and EDMS with no recurrent acute cerebrovascular events during the follow-up period. Angiographic evaluation indicated good revascularization and increased cerebral blood flow in most patients subjected to combined bypass. Collateral revascularization originating from EDMS was more predominant than that from STA-MCA anastomosis at the 6-month post-operative evaluation. The diameter of the major trunk of the STA, as well as its frontal and parietal branches increased profoundly at post-operative day 10 and had shrunk back to pre-operative size at 6 and 12 months, while the MMA and DTA demonstrated continuous enlargement during the follow-up time. No collateral discrepancies were observed 
Table II. Vascular reconstruction discrepancies at the postoperative 6-month evaluation between ischemic and hemorrhagic moyamoya patients.

\begin{tabular}{|c|c|c|c|c|c|c|}
\hline \multirow[b]{2}{*}{ Group } & \multirow[b]{2}{*}{ Hemispheres (n) } & \multicolumn{2}{|c|}{ Collateral grading on DSA } & \multicolumn{3}{|c|}{ Vascular reconstruction pattern } \\
\hline & & Score $0-1$ & Score 2-3 & Type I & Type II & Type III \\
\hline Ischemic & 49 & $3(6.12)$ & $46(93.88)$ & $12(24.49)$ & $17(34.69)$ & $20(40.82)$ \\
\hline Hemorrhagic & 17 & $1(5.88)$ & $16(94.12)$ & $4(23.53)$ & $6(35.29)$ & 7 (41.18) \\
\hline P-value & & 0.729 & & 1.000 & & \\
\hline
\end{tabular}

Values are expressed as n (\%). DSA, digital subtraction angiography.

between ischemic and hemorrhagic groups. The present study indicated that combined STA-MCA bypass and EDMS was a good option for treating MMD.

Intracranial and extracranial vascular reconstruction is the mainstay for MMD treatment $(1,2)$. Intracranial and extracranial vascular reconstruction surgery mainly includes direct and indirect revascularization. Direct revascularization increases the cerebral blood flow and instantly improves cerebral metabolism $(14,15)$, but the drawback is that STA or MCA branches of moyamoya patients may be fragile and easily occluded; thus, the long-term effect of direct revascularization remains elusive. In addition, patients with direct revascularization are prone to developing cerebral hemodynamic disturbances and perioperative complications (16). In addition, recipient vessels of MMD patients always have a small diameter and thin wall, which are difficult to be anastomosed by inexperienced surgeons or hospitals $(17,18)$. By contrast, indirect revascularization facilitates the formation of new blood vessels on the surface of the brain through the STA, the dura, the temporal muscle and the periosteum anastomosis (19-21). It has several merits, including safe and simple operation, as well as no damage to the already formed collateral circulation. However, certain studies reported that indirect revascularization is effective for children with MMD, but less effective for adults $(22,23)$. However, certain studies indicate that MMA and DTA may develop good neovasculature after indirect bypass, which is beneficial for alleviating clinical symptoms and stroke prevention $(24,25)$. In the present study, the reasons for poor collateral formation from indirect bypass may include the following: i) The bone flap is too small to make full use of the temporal muscle; ii) failure to maintain the integrity of the deep temporal artery when stripping the temporalis from the skull; iii) damage of the branches of the middle meningeal branch when milling of the bone flap or cutting of the dura mater. Therefore, in order to make full use of the DTA and MMA, a large frontal-temporal bone flap was generated to ensure that the temporal muscle was sufficiently attached to the surface of the brain. In addition, the arachnoid was fully opened to facilitate the contact between temporal muscle and cerebral cortex, as well as stimulate angiogenesis. Reverse temporal muscle separation technology was applied in our group to ensure the integrity of the deep temporal artery network, which allowed for maximum temporal muscle and cerebral cortex formation after anastomosis. The integrity of the MMA and its major branches was maintained without destroying already formed anastomosis between the MMA and cerebral cortex. The dura was reversed and stuck onto the surface of the cortex, which made the spontaneous anastomosis extend from the existing bone window. Overall, several surgical modifications may help moyamoya patients to form good collateral and develop optimal clinical outcomes.

In the present study, STA-MCA anastomosis was the treatment providing the most collateral formation in 19 hemispheres, which was less than the number of hemispheres in which EDMS had the predominant effect $(n=31)$. An equivalent contribution to revascularization by STA-MCA and EDMS was present in 25 hemispheres. These data suggested that EDMS and STA-MCA may contribute to revascularization corporately. According to the histopathological concepts, revascularization may include two types: Arteriogenesis and angiogenesis. Arteriogenesis is designated as the remodeling of pre-existing arterioarteriolar anastomoses, which may have a critical role in direct anastomosis $(13,26)$. Furthermore, the pressure gradient from the STA to the recipient artery is a key factor for promoting satisfactory arteriogenesis. Angiogenesis usually occurs in the fibrous coat developing between the transplanted muscle and the arachnoid membrane $(13,27)$, which is assumed to participate in indirect anastomosis. To this point, there also exits a reciprocal mechanism to develop completed collaterations between direct STA-MCA bypass and indirect EDMS.

Previous studies reported that STA-MCA bypass increases STA and MCA blood flow during the early vascular remodeling period $(14,15)$. The increase of STA blood flow may be associated with the low resistance of the intracranial vascular bed, while the increase of MCA blood flow may be due to the support of the STA. Consistent with this, the present study observed that the STA was enlarged during the early post-operative phase and shrank gradually, while the MMA and DTA demonstrated continuous widening during the follow-up period. With the development of indirect revascularization and enlargement of MMA and DTA, the blood flow demand from the STA-MCA declines $(28,29)$, which is a possible reason for the thinning and even occlusion of the STA. These alterations suggested that direct STA-MCA bypass provides early augmentation of cerebral perfusion, whereas indirect EDMS provides a more durable long-term revascularization.

Therefore, the present study suggests that combining direct and indirect bypass in a suitable effort to take 
advantage of the immediate revascularization provided by direct arterial bypass, but also to maximize the eventual cerebral revascularization.

\section{Acknowledgements}

The authors would like to thank Dr Meijuan Zhang (Department of Neurology, The Affiliated Drum Tower Hospital of Nanjing University, Nanjing, China) for professional language editing of the manuscript. This study was supported by the National Natural Science Foundation of China (grant no. 81301049), the Natural Science Foundation of Jiangsu Province of China (grant no. BK20130085) and the Project supported by the Medical Science and Technology Development Foundation, Nanjing Department of Health (grant no. YKK14100). Part of this study was previously presented at the International Stroke Conference, February 2017, poster no. TP449.

\section{Competing interests}

The authors declare that they have no competing interests.

\section{References}

1. Pandey P and Steinberg GK: Neurosurgical advances in the treatment of moyamoya disease. Stroke 42: 3304-3310, 2011.

2. Scott RM and Smith ER: Moyamoya disease and moyamoya syndrome. N Engl J Med 360: 1226-1237, 2009.

3. Arias EJ, Derdeyn CP, Dacey RG Jr and Zipfel GJ: Advances and surgical considerations in the treatment of moyamoya disease. Neurosurgery 74 (Suppl 1): S116-S125, 2014.

4. Baaj AA, Agazzi S, Sayed ZA, Toledo M, Spetzler RF and van Loveren H: Surgical management of moyamoya disease: A review. Neurosurg Focus 26: E7, 2009.

5. Houkin K, Ishikawa T, Yoshimoto T and Abe H: Direct and indirect revascularization for moyamoya disease surgical techniques and peri-operative complications. Clin Neurol Neurosurg 99 (Suppl 2): S142-S145, 1997.

6. Kim DS, Huh PW, Kim HS, Kim IS, Choi S, Mok JH and Huh CW: Surgical treatment of moyamoya disease in adults: Combined direct and indirect vs. indirect bypass surgery. Neurol Med Chir 52: 333-338, 2012.

7. Czabanka M, Peña-Tapia P, Scharf J, Schubert GA, Munch E, Horn P, Schmiedek P and Vajkoczy P: Characterization of direct and indirect cerebral revascularization for the treatment of european patients with moyamoya disease. Cerebrovasc Dis 32 : 361-369, 2011.

8. Rashad S, Fujimura M, Niizuma K, Endo H and Tominaga T: Long-term follow-up of pediatric moyamoya disease treated by combined direct-indirect revascularization surgery: Single institute experience with surgical and perioperative management. Neurosurg Rev 39: 615-623, 2016.

9. Oikawa S, Mizuno M, Muraoka S and Kobayashi S: Retrograde dissection of the temporalis muscle preventing muscle atrophy for pterional craniotomy. Technical note. J Neurosurg 84: 297-299, 1996

10. Kadri PA and Al-Mefty O: The anatomical basis for surgical preservation of temporal muscle. J Neurosurg 100: 517-522, 2004

11. Suzuki J and Takaku A: Cerebrovascular 'moyamoya' disease. Disease showing abnormal net-like vessels in base of brain. Arch Neurol 20: 288-299, 1969.
12. Matsushima T, Inoue T, Suzuki SO, Fujii K, Fukui M and Hasuo K: Surgical treatment of moyamoya disease in pediatric patients-comparison between the results of indirect and direct revascularization procedures. Neurosurgery 31: 401-405, 1992.

13. Saito $\mathbf{N}$ and Imai H: Insights on the revascularization mechanism for treatment of moyamoya disease based on the histopathologic concept of angiogenesis and arteriogenesis. World Neurosurg 75: 204-205, 2011.

14. Lee M, Guzman R, Bell-Stephens $\mathrm{T}$ and Steinberg GK: Intraoperative blood flow analysis of direct revascularization procedures in patients with moyamoya disease. J Cereb Blood Flow Metab 31: 262-274, 2011.

15. Arikan F, Vilalta J, Torne R, Noguer M, Lorenzo-Bosquet C and Sahuquillo J: Rapid resolution of brain ischemic hypoxia after cerebral revascularization in moyamoya disease. Neurosurgery 76 : 302-312, 2015.

16. Yu J, Shi L, Guo Y, Xu B and Xu K: Progress on complications of direct bypass for moyamoya disease. Int J Med Sci 13: 578-587, 2016.

17. Takagi Y, Hermanto Y, Takahashi JC, Funaki T, Kikuchi T, Mineharu Y, Yoshida K and Miyamoto S: Histopathological characteristics of distal middle cerebral artery in adult and pediatric patients with moyamoya disease. Neurol Med Chir (Tokyo) 56: 345-349, 2016.

18. Lin R, Xie Z, Zhang J, Xu H, Su H, Tan X, Tian D and Su M: Clinical and immunopathological features of moyamoya disease. PLoS One 7: e36386, 2012.

19. Kuroda S and Houkin K: Moyamoya disease: Current concepts and future perspectives. Lancet Neurol 7: 1056-1066, 2008.

20. Scott RM: Surgery for moyamoya syndrome? Arch Neurol 58: 128-129, 2001.

21. Zipfel GJ, Fox DJ Jr and Rivet DJ: Moyamoya disease in adults: The role of cerebral revascularization. Skull Base 15:27-41, 2005.

22. Macyszyn L, Attiah M, Ma TS, Ali Z, Faught R, Hossain A, Man K, Patel H, Sobota R, Zager EL and Stein SC: Direct versus indirect revascularization procedures for moyamoya disease: A comparative effectiveness study. J Neurosurg 126: 1523-1529, 2017.

23. Mizoi K, Kayama T, Yoshimoto T and Nagamine Y: Indirect revascularization for moyamoya disease: Is there a beneficial effect for adult patients? Surg Neurol 45: 541-549, 1996.

24. Kinugasa K, Mandai S, Kamata I, Sugiu K and Ohmoto T: Surgical treatment of moyamoya disease: Operative technique for encephalo-duro-arterio-myo-synangiosis, its follow-up, clinical results, and angiograms. Neurosurgery 32: 527-531, 1993.

25. Uchino H, Kim JH, Fujima N, Kazumata K, Ito M, Nakayama N, Kuroda S and Houkin K: Synergistic interactions between direct and indirect bypasses in combined procedures: The significance of indirect bypasses in moyamoya disease. Neurosurgery 80: 201-209, 2017.

26. Heil $\mathrm{M}$ and Schaper $\mathrm{W}$ : Insights into pathways of arteriogenesis. Curr Pharm Biotechnol 8: 35-42, 2007.

27. Nakamura M, Imai H, Konno K, Kubota C, Seki K, Puentes S, Faried A, Yokoo H, Hata H, Yoshimoto Y and Saito N: Experimental investigation of encephalomyosynangiosis using gyrencephalic brain of the miniature pig: Histopathological evaluation of dynamic reconstruction of vessels for functional anastomosis. Laboratory investigation. J Neurosurg Pediatr 3: 488-495, 2009.

28. King JA, Armstrong D, Vachhrajani S and Dirks PB: Relative contributions of the middle meningeal artery and superficial temporal artery in revascularization surgery for moyamoya syndrome in children: The results of superselective angiography. J Neurosurg Pediatr 5: 184-189, 2010.

29. Amin-Hanjani S, Singh A, Rifai H, Thulborn KR, Alaraj A, Aletich V and Charbel FT: Combined direct and indirect bypass for moyamoya: Quantitative assessment of direct bypass flow over time. Neurosurgery 73: 962-967, 2013. 